\title{
Genome adaptive evolution of Lactobacillus casei under long-term antibiotic selection pressures
}

Jicheng Wang ${ }^{1,2 \dagger}$, Xiao Dong ${ }^{1,2+}$, Yuyu Shao ${ }^{1,2}$, Huiling Guo ${ }^{1,2}$, Lin Pan ${ }^{1,2}$, Wenyan Hui ${ }^{1,2}$, Lai-Yu Kwok ${ }^{1,2}$, Heping Zhang ${ }^{1,2}$ and Wenyi Zhang ${ }^{1,2^{*}}$

\begin{abstract}
Background: The extensive use of antibiotics in medicine has raised serious concerns about biosafety. However, the effect of antibiotic application on the adaptive evolution of microorganisms, especially to probiotic bacteria, has not been well characterized. Thus, the objective of the current work was to investigate how antibiotic selection forces might drive genome adaptation using Lactobacillus (L.) casei Zhang as a model.

Methods: Two antibiotics, amoxicillin and gentamicin, were consistently applied to the laboratory culture of L. casei Zhang. We then monitored the mutations in the bacterial genome and changes in the minimum inhibitory concentrations (MICS) of these two antibiotics along a 2000-generation-cultivation lasted over 10 months.

Results: We found an approximately 4 -fold increase in the genome mutation frequency of L. casei Zhang, i.e. $3.5 \times$ $10^{-9}$ per base pair per generation under either amoxicillin or gentamicin stress, when compared with the parallel controls grown without application of any antibiotics. The increase in mutation frequency is significantly lower than that previously reported in Escherichia (E.) coli. The rate of de novo mutations, i.e. 20 per genome, remained low and stable throughout the long-term cultivation. Moreover, the accumulation of new mutations stopped shortly after the maximum bacterial fitness (i.e. the antibiotic MICS) was reached.

Conclusions: Our study has shown that the probiotic species, L. casei Zhang, has high genome stability even in the presence of long-term antibiotic stresses. However, whether this is a species-specific or universal characteristic for all probiotic bacteria remains to be explored.
\end{abstract}

Keywords: Lactobacillus casei Zhang, Amoxicillin, Gentamicin, Biosafety

\section{Background}

The evolution of antibiotic resistance in bacteria has long been an important issue because of the wide application of antibiotics in medicine [1]. The recently developed next generation sequencing technology has greatly improved the process of DNA sequencing. It has also become an affordable tool for characterizing bacterial genome evolution by monitoring the genome changes along long-term laboratory growth. Such approach,

\footnotetext{
* Correspondence: zhangwenyizi@163.com

${ }^{\dagger}$ Equal contributors

'Key Laboratory of Dairy Biotechnology and Engineering, Ministry of Education, Inner Mongolia Agricultural University, Hohhot, Inner Mongolia 010018, China

${ }^{2}$ Key Laboratory of Dairy Products Processing, Ministry of Agriculture, Inner Mongolia Agricultural University, Hohhot 010018, Inner Mongolia, China
}

adaptive laboratory evolution (ALE), is used for analyzing the evolutionary phenomena of bacteria in a controlled laboratory setting; and it has revealed new insights into microbial genetic adaptations under certain culture conditions [2]. The first description of ALE experiments can be dated back to the study published by Russel in 1893 [3]. Currently, most studies concerning phenotypic and genotypic changes in adaptive evolution are limited to the species Escherichia (E.) coli [4].

The genus Lactobacillus is a major part of the lactic acid bacteria (LAB) group that consists of more than 200 known species and subspecies [5]. Because of its ability to ferment raw materials, Lactobacillus has a long history of safe use in food production, such as yogurt, cheese, beer, wine, and other fermented foods. Our 
research team has previously established a gene catalogue of Lactobacillus by comparative genomics analysis. Particularly, our work has described a broad and diverse carbohydrate- and protein-modifying genes, as well as the presence of multiple novel CRISPR-Cas immune systems. Moreover, our data revealed how the Lactobacillus host interaction factors and bacteriocins affect their natural and industrial environments, and the mechanisms of these bacteria in withstanding stress during technological processes [6]. Apart from being used as starter bacteria in food fermentation, Lactobacillus strains that have known health-promoting effects are also applied as probiotics [7]. These strains are widely used in functional fermented dairy products, veterinary medicines, and feed additives $[8,9]$.

Recently, there are growing interests in the combined use of probiotics and antibiotics to treat gastrointestinal disorders [10]. One major mechanism that renders previously non-resistant bacteria to become resistant to a particular antibiotic is the selection of mutated subpopulations that carry obliterated antibiotic target genes or have acquired novel abilities to remove or deactivate the effective drug [11]. Thus, frequent exposure to an antibiotic-containing environment may make the probiotics more prone to gain additional antibiotic resistance capacity. These bacteria have to reach and be present at least temporarily in the intestines of humans and animals to exert their beneficial effects. During their transit through the host gastrointestinal tract, the mutated antibiotic resistance genes will be directly exposed to other co-occurring gut microbes [12]. Meanwhile, there is an increased chance of interaction between the probiotic bacteria and the complex colon gut microbial community. Despite the potential risk, the evolutionary adaptation of probiotics towards antibiotic resistance has not been adequately studied. Only one work regarding this topic has been published up to now, which characterized the spontaneous drug resistance of Lactobacillus at the biochemical level [13].

Therefore, the objective of the current work was to investigate how antibiotic selection forces might drive the genome adaptation in Lactobacillus (L.) casei Zhang. Lactobacillus casei Zhang is a probiotic strain isolated from koumiss. It is highly resistant to acid and bile stresses; and it exhibits anti-bacterial and anti-oxidative properties $[14,15]$. One obvious advantage of using $L$. casei Zhang as a model is the availability of the detailed genome sequence information. The genome of $L$. casei Zhang consists of a circular chromosome $(2,861,848$ base pair, bp) and a single plasmid (36 kilobase, kb) [1618]. The information serves as a reference to identify any mutations and genomic changes. Our study monitored the genotypic and phenotypic changes of $L$. casei Zhang along a 2000-generation-cultivation lasted over
10 months under three growth conditions with or without antibiotics (amoxicillin or gentamicin). To our knowledge, this is the first report investigating the antibiotic-driven genome adaptation of a common food use LAB.

\section{Methods}

\section{Bacterial growth conditions}

The bacterium, $L$. casei Zhang, was used in the current study. It was grown either in de Mann-Rogosa-Sharpe (MRS) broth (CM0359,OXOID) or LAB susceptibility test medium (LSM) [19]. The LSM was made of 90\% Isosensitest medium (IST; CM0473, OXOID) and 10\% MRS.

\section{Experimental evolution}

The laboratory evolution experiments were designed based on the standard protocol described by Pena-Miller et al. [20]. Briefly, an original stock of $L$. casei Zhang was subcultured twice in MRS broth. Then they were streaked onto MRS agar plates. After cultivating at $37^{\circ} \mathrm{C}$ for $72 \mathrm{~h}$, three colonies were randomly picked and were separately inoculated into three tubes of $5 \mathrm{~mL}$ LSM supplemented with gentamicin $(1 \mu \mathrm{g} / \mathrm{mL})$ (strain G), amoxicillin $(0.5 \mu \mathrm{g} / \mathrm{mL})$ (strain A), or without any antibiotics (strain C). The antibiotic concentrations used were the half maximum inhibitory concentrations $\left(\mathrm{IC}_{50}\right)$ of $L$. casei Zhang determined previously (unpublished data). Each culture was continuously propagated by transferring $1 \%(\mathrm{v} / \mathrm{v}, 50 \mu \mathrm{L})$ of the original culture into fresh medium every $24 \mathrm{~h}$. Approximately, 100-fold daily bacterial growth represented nearly 6.6 generations per subculture. Samples were collected for fitness evaluation and identification of de novo mutations every 200 generations. Frozen stocks were prepared regularly along the long-term cultivation. Strains were numbered in consecutive order according to their generations.

\section{Evaluation of fitness as represented by minimum inhibitory concentrations (MICs)}

The phenotypic changes of the bacterial cultures were measured by the gentamicin and amoxicillin MICs, which were determined using the broth macrodilution method described by Shao et al. [21]. Briefly, bacterial suspensions with turbidity equivalent to McFarland standard $1\left(\sim 3 \times 10^{8} \mathrm{cfu} / \mathrm{mL}\right)$ were prepared. They were then diluted by 500 folds $\left(\sim 6 \times 10^{5} \mathrm{cfu} / \mathrm{mL}\right)$. Doubling dilutions of antibiotics, ranging from 0.25 to $256 \mu \mathrm{g} / \mathrm{ml}$ for both gentamicin and amoxicillin, were freshly prepared in LSM broth. Each antibiotic-containing or control tube was then inoculated with an equal volume of the respective diluted bacterial suspension (i.e. a final bacterial concentration of $3 \times 10^{5} \mathrm{cfu} / \mathrm{mL}$ ). The MIC endpoints were read after $48 \mathrm{~h}$ incubation at $37{ }^{\circ} \mathrm{C}$ under 
strictly anaerobic conditions. The assay was repeated three times.

\section{Identification of de novo mutations}

The adapted strains were cultured under anaerobic conditions in LSM broth at $37^{\circ} \mathrm{C}$, followed by DNA extraction with a bacterial DNA extraction kit (OMEGA D3350-02) according to the manufacturer's instructions. Genomic DNA samples were quantified by using a TBS380 fluorometer (Turner BioSystems Inc., Sunnyvale, CA). The DNA quality was checked by spectrophotometry. Qualified DNA samples $\left(\mathrm{OD}_{260 / 280}=1.8 \sim 2.0\right.$, > 6ug) were utilized to construct libraries of 200 to $300 \mathrm{bp}$ fragments.

We selected and sequenced 50 genomes at generations 200, 400, 600, 800, 1000, 1200, 1400, 1800, and 2000. For all sequencing, at least $3 \mu \mathrm{g}$ genomic DNA was used for sequencing library construction. Paired-end libraries with an insert size of $\sim 300 \mathrm{bp}$ were prepared following the Illumina's standard genomic DNA library preparation procedures. Purified genomic DNA was sheared into smaller fragments to a desirable size using Covaris fragmentation, and blunt ends were generated using T4 DNA polymerase. After adding an ' $\mathrm{A}$ ' base to the 3' end of the phosphorylated DNA fragments, adapters were ligated to the ends. The desired fragments were purified by agarose gel electrophoresis before being selectively enriched and amplified by polymerase chain reaction (PCR). An index tag was introduced via an adapter during PCR. A library quality test was then performed, and the qualified Illumina pair-end library was used for Illumina Hiseq 2000 (Illumina Inc. U.S.A) sequencing. The read lengths were $100 \mathrm{bp}$. An average of $617.92 \mathrm{Mb}$ of high-quality data were generated for each strain, corresponding to a sequencing depth of 157 to 282 folds.

For variant calling, the raw sequence reads were imported into CLC Bio Genomics Workbench V8.5.1 (CLC Inc., Aarhus, Denmark) and mapped against the $L$. casei Zhang genome with $80 \%$ identity and a length fraction setting of 0.5. Two built-in variant detection tools, namely Fixed Ploidy Variant Detection and Low Frequency Variant Detection, were used to call mutations. The parameters of the former detector were single nucleotide variation (SNV) coverage of $>20$, variant probability of $90 \%$, and ploidy set at 1 (high-quality call set), whilst the parameters of the latter detector were the same level of SNV coverage, minimum frequency of $1 \%$, and significance of $1 \%$ (lenient call set). Synonymous or non-synonymous sites were discriminated by using the protocol of Amino Acid Changes available in the Functional Consequences module.

To identify true de novo mutations that occurred during the long-term cultivation, the common SNVs shared by multiple strains in the high-quality call set were filtered out, as these might have been natural SNVs preexistent before the experiment. To track when the de novo mutations happened and their stability, the lenient call set was analyzed. For each reliable de novo mutation identified, we determined the generations of its first and last presence along the adaptive evolution experiment. $\mathrm{Ka} / \mathrm{Ks}$ ratio was calculated by dividing the number of non-synonymous substitutions per non-synonymous site (Ka) by the number of synonymous substitutions per synonymous site (Ks) [22]. The L. casei codon usage and its frequency were obtained from Kazusa DNA Research Institute (KDRI, http://www.kazusa.or.jp/codon/).

To verify the identified variants, a set of reliable SNVs was randomly selected and sequenced by Sanger method. Sequencing of the amplicons was performed by Shanghai Majorbio Bio-Pharm Technology Co., Ltd.

\section{Results}

Long-term evolution of $L$. casei Zhang with and without antibiotic exposure

We monitored the evolution of $L$. casei Zhang along a 2000-generation-cultivation under three experimental conditions, i.e. with amoxicillin (strain A) or gentamicin (strain G), and control without any antibiotics (strain C). We assessed the strain fitness by monitoring the changes in the amoxicillin and gentamicin MICs at different time points in each case. At generation 0, the MICs for amoxicillin and gentamicin were the same $(2 \mu \mathrm{g} / \mathrm{mL})$. The amoxicillin MIC for strain A increased to a maximum level $(8 \mu \mathrm{g} / \mathrm{mL})$ after subculturing for 400 generations, while the gentamicin MIC for strain G reached 32 $\mu \mathrm{g} / \mathrm{mL}$ after subculturing for 1200 generations (Tables 1 and 2). The MIC of the control, strain C, remained unchanged. This indicates that strains $A$ and $G$ had

Table 1 Changes in gentamicin minimum inhibitory concentration (MIC) of Lactobacillus casei Zhang during long-term cultivation in lactic acid bacteria susceptibility test medium (LSM) with gentamicin $(\mu \mathrm{g} / \mathrm{mL})$

\begin{tabular}{llll}
\hline Generation & \multicolumn{2}{l}{ MIC (Gentamicin $\mu \mathrm{g} / \mathrm{mL})$} & $\mathrm{G} 3$ \\
\cline { 2 - 4 } & $\mathrm{G} 1$ & $\mathrm{G} 2$ & 2 \\
\hline 0 & 2 & 8 & 4 \\
200 & 8 & 8 & 8 \\
400 & 8 & 8 & 8 \\
600 & 8 & 16 & 16 \\
800 & 16 & 16 & 16 \\
1000 & 16 & 32 & 32 \\
1200 & 32 & 32 & 32 \\
1400 & 32 & 32 & 32 \\
1600 & 32 & 32 & 32 \\
1800 & 32 & 32 & 32 \\
2000 & 32 & &
\end{tabular}


Table 2 Changes in amoxicillin minimum inhibitory concentration (MIC) of Lactobacillus casei Zhang during longterm cultivation in lactic acid bacteria susceptibility test medium (LSM) with amoxicillin $(\mu \mathrm{g} / \mathrm{mL})$

\begin{tabular}{llll}
\hline Generation & \multicolumn{2}{l}{ MIC (Amoxicillin $\mu \mathrm{g} / \mathrm{mL})$} & $\mathrm{A} 3$ \\
\cline { 2 - 4 } & $\mathrm{A} 1$ & 2 & 2 \\
\hline 0 & 2 & 4 & 4 \\
200 & 4 & 8 & 4 \\
400 & 4 & 8 & 8 \\
600 & 8 & 8 & 8 \\
800 & 8 & 8 & 8 \\
1000 & 8 & 8 & 8 \\
1200 & 8 & 8 & 8 \\
1400 & 8 & 8 & 8 \\
1600 & 8 & 8 & 8 \\
1800 & 8 & 8 & 8 \\
2000 & 8 & &
\end{tabular}

increased fitness towards amoxicillin and gentamicin respective to the exposed antibiotics. The rate of adaptation to amoxicillin was about three-fold higher than that of gentamicin.

\section{Accumulation of de novo mutations}

For every 100 generations, frozen stocks of strains A, G, and $C$ were prepared. They were recovered at the end of the experiment and were sequenced. De novo mutations were identified by comparing the obtained sequences with the reference genome of $L$. casei Zhang [17]. Possibly, due to the physical damage exerted to the bacteria during frozen stock preparation, a few of the samples for certain time points (from culture conditions of C3, G2, A1, and A2) could not be recovered or grew poorly after being thawed. We therefore excluded these problematic samples in the genomic analysis. Fortunately, representative samples were obtained to cover all time points along the 10-month experiment, so that a complete image of evolution could be presented.

We randomly selected 11 mutations and performed Sanger sequencing to validate mutation calling. Every SNV-containing locus was sequenced from both directions, covering approximately $400 \mathrm{bp}$. Nine out of the 11 variants found by Sanger sequencing were validated, which is comparable to a previous study [23]. The complete list of the confirmed SNVs with the respective predicted changes at amino acid level is provided in Additional file 1: Table S1. Such results validated our sequencing and mutation calling methodologies in identifying de novo mutations. The mutations identified were distributed to a high proportion of the sequence reads. Since we excluded the common SNPs and identified mutations shared across strains $A, G$, and $C$ in subsequent analysis, the accumulated de novo mutations found in this study truly reflected the genomic changes happened during the long-term propagation. These de novo mutations were regarded as fixed variants. We traced these de novo mutations in the lenient criteria call set and identified when they first occurred.

The accumulation of de novo mutations along the long-term cultivation is presented in Fig. 1. We observed an around 4-fold increase in mutation accumulation (including SNVs, short insertions and deletions) in both strains A and G, comparing to the control (strain C) (i.e. an average of 19,21.5, and 5.5 de novo mutations for strains A, G, and C, respectively). These corresponded to 0.01 de novo mutation per generation $\left(3.5 \times 10^{-9}\right.$ per base pair per generation) under the amoxicillin or gentamicin selection pressure, comparing to 0.003 de novo mutation per generation $\left(1.0 \times 10^{-9}\right.$ per base pair per generation) for the control. Such results are suggestive of apparent adaptive evolution of the $L$. casei Zhang genome, particularly when grown in the presence of antibiotics.

In parallel with the changes in gentamicin and amoxicillin MICs, the de novo mutation accumulation reached the maximum level at generation 1200 in strain G (Fig. 1). The mutation accumulation rate of strain A was highest during the first 400 generations (Fig. 1). Mutation accumulation still continued at a relatively high rate until generation 1000 .

\section{Spectrum of de novo mutations}

We further compared the spectrum of de novo mutations occurred in the three strains. We focused on the de novo SNVs because of the low number of observed small insertions and deletions (i.e. 1 insertion and 3 deletions in strain G, 1 deletion in the control, and no insertion or deletion in strain A), which would be inadequate for drawing any valid conclusions.

As illustrated in Fig. 2, AT- > CG and CG->AT transversions were the two most common classes of de novo SNVs, contributing to $26.2 \%$ and $40.8 \%$ of the total detected mutations, respectively. Additionally, there was a high level of CG- > TA transition, i.e. $21.4 \%$ of the total detected mutations. No significant difference was observed between the mutation spectrum of either of the antibiotic exposed strains (strain A or G) and the control (strain $\mathrm{C})$. This indicates that, as expected, the amoxicillin or gentamicin stress did not drive any specific type of mutations. The transition to transversion ratio $(\mathrm{Ti} / \mathrm{Tv})$ was $0.45,0.33$, and 0.29 for strains $\mathrm{A}, \mathrm{G}$, and $\mathrm{C}$, correspondingly. Although the difference in $\mathrm{Ti} / \mathrm{Tv}$ between strains $\mathrm{A}$ and $\mathrm{C}$ was 0.16 , such difference could not be considered significant due to the limited number of identified mutations, especially in the control (strain C). 


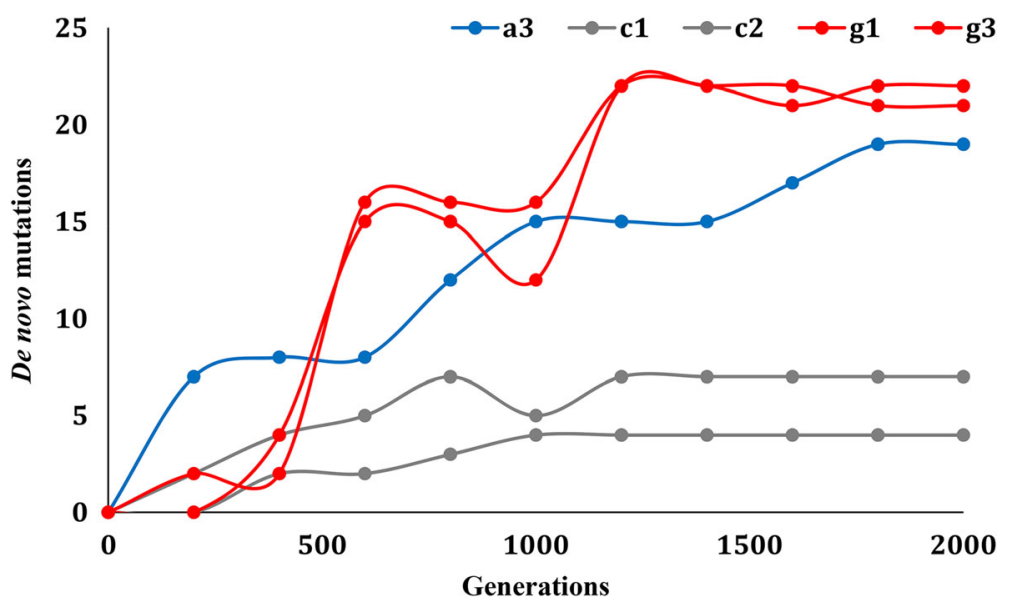

Fig. 1 Mutation accumulation of Lactobacillus casei Zhang along the long-term cultivation in lactic acid bacteria susceptibility test medium (LSM) with and without antibiotics

To demonstrate if positive selection had driven the evolution of strains A and G, we compared the fractions of non-synonymous mutations between strains with and without antibiotic treatment. We found significantly higher proportions of non-synonymous mutations in strains A (75\%) and G (69\%), comparing to the control (50\%) $(P=0.029$ and 0.049 , respectively, determined by one-tailed $\mathrm{z}$ test for two proportions). The $\mathrm{Ka} / \mathrm{Ks}$ ratio of strains A, G, and control were $1.02,0.94$, and 0.68 , respectively. This shows that the control was protected from functional mutations, while the mutations in strains A and G occurred almost neutrally driven by amoxicillin and gentamicin application, correspondingly.

\section{Functional evaluation of the de novo mutations}

Since non-synonymous mutations could possibly alter the functions of the coding genes, we assigned all the mutated genes into Clusters of Orthologous Groups (COGs) that classify genes based on functions. To estimate the number of mutated genes in different COG categories precisely, the same genes from the replicate cultures were considered as different genes and the number was accumulated. Common mutated genes that were shared across the amoxicillin, gentamicin, and control strains belonged mainly to two COG categories, transcription $[\mathrm{K}]$ and cell wall/membrane/envelope biogenesis [M] (Fig. 3). Compared to strains $\mathrm{C}$ and $\mathrm{A}$, strain $\mathrm{G}$ had a more unique set of mutated genes, which was related to energy production and conversion $[\mathrm{C}]$, amino acid transport and metabolism [E], lipid transport and metabolism, translation [I], ribosomal structure and biogenesis $[J]$, general function prediction only $[R]$, and defense mechanisms [V].

Specifically, a Xenobiotic Response Element (XRE) family transcriptional regulator (LCAZH_1179) was found mutated in both strains A and G. The XRE family transcription factors are the second most frequently occurring regulator family among bacteria that participate in diverse metabolic functions [24]. Other putative genes that are involved in gene regulation included some transcriptional regulators (LCAZH_1767, LCAZH_1064, LCAZH_0070, and LCAZH_0059), some OmpR family

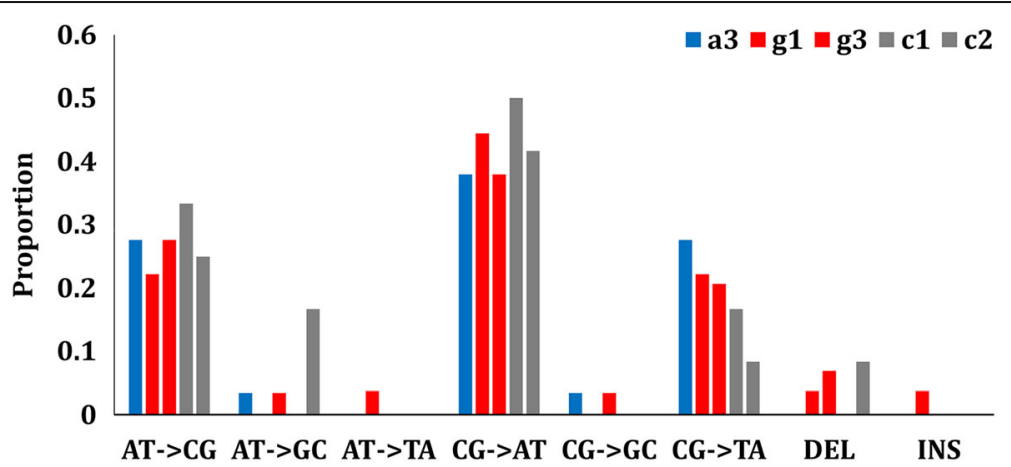

Fig. 2 Spectrum of accumulated mutations of Lactobacillus casei Zhang along the long-term cultivation in lactic acid bacteria susceptibility test medium (LSM) with and without antibiotics 


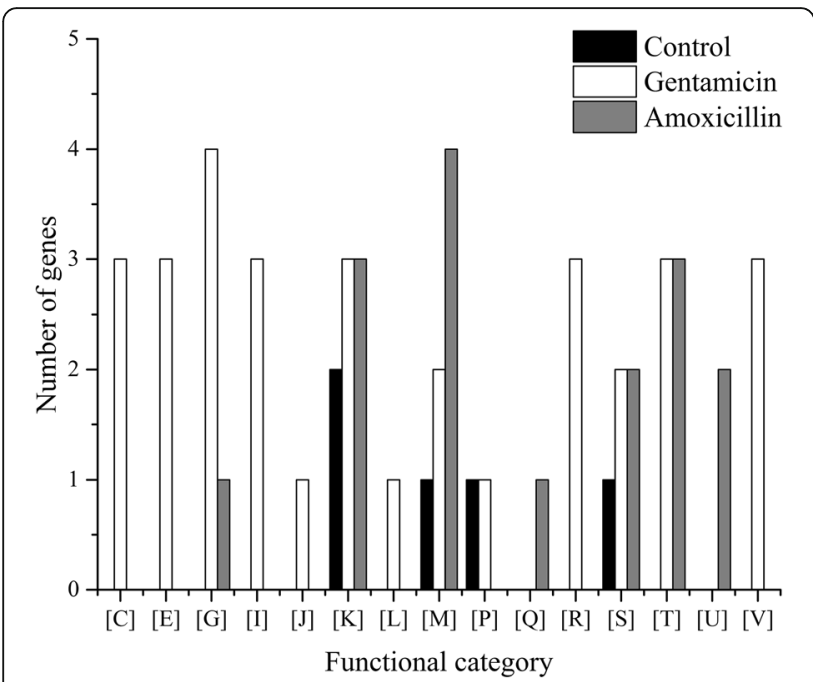

Fig. 3 Clusters of orthologous groups (COG) of mutated genes of Lactobacillus casei Zhang during long-term cultivation in lactic acid bacteria susceptibility test medium (LSM) with and without antibiotics. Functional categories: [C], Energy production and conversion; [E], Amino acid transport and metabolism; [F], Nucleotide transport and metabolism; [G], Carbohydrate transport and metabolism; [H], Coenzyme transport and metabolism; [1], Lipid transport and metabolism; []], Translation, ribosomal structure and biogenesis; [K], Transcription; [L], Replication, recombination and repair; [M], Cell wall/membrane/envelope biogenesis; [O], Posttranslational modification, protein turnover, chaperones; $[\mathrm{P}]$, Inorganic ion transport and metabolism; [Q], Secondary metabolites biosynthesis, transport and catabolism; [R], General function prediction only; [S], Function unknown; [T], Signal transduction mechanisms; IV], Defense mechanisms

DNA-binding response regulators (LCAZH_1669 and LCAZH_0490), a signal transduction histidine kinase (LCAZH_1668), a serine/threonine protein phosphatase (LCAZH_1613), a sensor histidine kinase PrcK (LCAZH_2350), and a DNA-directed RNA polymerase subunit beta (LCAZH_2481). Among them, the regulators of OmpR family is directly associated with antibiotic, extreme $\mathrm{pH}$, temperature, and oxidative stresses [25]. Both of their coding proteins contain an Nterminal receiver (IPR001789) and a C-terminal DNAbinding domain (IPR001789).

Moreover, antibiotic-driven mutations were found in several other genes that are potentially associated with stress responses. Membrane modification is one of the key mechanisms of bacterial bile tolerance [26]. We have identified de novo mutations in seven genes that code for proteins relating to cell surface features, including a membrane carboxypeptidase (LCAZH_1472), a glycosyltransferase (LCAZH_2870), a mismatch repair ATPase (LCAZH_0590), a Dalanine-activating enzyme (LCAZH_0737), a cyclopropane fatty acid synthase-like methyltransferase (LCAZH_2067), and two FtsI/penicillin-binding proteins (LCAZH_1260 and LCAZH_1652). According to Denome et al. [27], three of these genes code for proteins (LCAZH_1472, LCAZH_1260, and LCAZH_1652) belonging to the penicillin-binding protein family that plays a role in the biosynthesis of cell wall peptidoglycan. Some members of this family act like glycosyltransferases, which are required for glycan chain polymerization [28]. Indeed, membrane modification has also been observed when bacteria are under acid stress. For example, when $L$. casei was adapting to an acidic environment, a cyclopropane fatty acid synthase-like methyltransferase was activated and expressed constitutively [29]. The enzyme catalyzes the addition of a methylene residue across the cis double bond of $C_{16: \ln (9)}, C_{18: \ln (9)}$, or $C_{18: \ln (11)}$ unsaturated fatty acids to form an unsaturated cyclopropane derivative. Another example is the D-alanine-activating enzyme, which was found to be associated with acid tolerance of Streptococcus mutans [30]. This enzyme catalyzes the first step of teichoic acids synthesis.

In response to external stresses, bacteria may modulate their energy metabolism. We located de novo mutations in the ATP synthase F0F1 subunits (LCAZH_1154 and LCAZH_1156), which are responsible for energy production and proton extrusion. Proton extrusion is also recognized as a mechanism of acid tolerance in bacteria [31].

\section{Discussion}

Previously, Curragh and Collins reported a high spontaneous mutation frequency in Lactobacillus when the bacteria were cultivated with antibiotics, including nitrofurazone, kanamycin, and streptomycin; meanwhile, the bacterial antibiotic resistance increased together with the high spontaneous mutation frequency [13]. Similarly, we found an increased mutation frequency, i.e. 4-fold higher, in strains grown in an amoxicillin- or gentamicin-containing environment, comparing to the control strain without antibiotic treatment, after subculturing for 2000 generations. Most of these mutations occurred before the first 1200 generations with a significantly higher proportion of non-synonymous mutations. Based on our results, the non-synonymous mutations were mostly found in genes involving in gene regulation and stress responses; thus, it is likely that they were function-driven, and they potentially contributed to the bacterial fitness adaptation. It is known that genetic changes may result in additional antibiotic resistance to pathogens that are not intrinsically present via various mechanisms, e.g. acquiring efflux system coding genes that pump out the antibiotic molecules, altering the antibiotics target enzymes, and degrading/modifying antibiotic molecules [32]. Whether the de novo mutations seen in our study confer additional resistance capacity to the strains cultivated with antibiotics remains to be determined in future studies. 
The natural spontaneous base pair substitution (fixed) rate of $E$. coli was $\sim 5$-fold lower than that observed in $L$. casei Zhang (1.6 to $2.2 \times 10^{-10}$ versus $1.0 \times 10^{-9}$ per bp per generation for $E$. coli and $L$. casei Zhang, respectively) $[2,33]$. However, under antibiotic stresses, the mutation frequency of $L$. casei Zhang was only one-third of that of E. coli $\left(3.5 \times 10^{-9}\right.$ versus $1.1 \times 10^{-8}$ per bp per generation) [2]. This suggests that although the genome of $L$. casei Zhang is less stable than that of $E$. coli under normal conditions, it may respond less to antibiotic stress. Further side-by-side comparative studies of different species are required to reach a more definite conclusion regarding whether high genome stability is a species-specific or universal characteristic for all probiotic bacteria.

A higher proportion of CG- > AT transversion was found in L. casei Zhang (40.8\%; Fig. 2) compared to $E$. coli $(23.5 \%)$ [2], even though the GC content is lower in the genome of $L$. casei Zhang $(46.4 \%$ versus $50.6 \%$ in $E$. coli). The mutation spectrum of $L$. casei Zhang was similar, whether antibiotics were applied or not. Even without exposure to any external mutagen, $L$. casei Zhang exhibited a relatively high CG-> AT transversion rate. This may be a natural characteristic specific to this species due to a possible lack of the AG- or CT-mispair correction capacity [34].

Some pathogens have been shown to adapt rapidly upon antibiotic exposure by continuous accumulation of point mutations and thus raise serious health and safety concerns to the public [11]. However, unlike in the cases of pathogens, no significant increase in both mutation accumulation and MIC values was observed in $L$. casei Zhang in the later generations even with continuous antibiotic exposure. Instead, the mutation rate returned to the baseline level similar to that of the control, suggesting that both the bacterial genome and fitness level to antibiotics were stabilized. Our findings show that $L$. casei Zhang is a stable strain and presents limited biosafety risks even subject to long-term antibiotic exposure. However, the scope of the current study is limited to the analysis of mutation accumulation under in vitro cultivation conditions. Another important mechanism that drives evolution is horizontal gene transfer, which has not been addressed here. Particularly, when the probiotic bacteria arrive in the host gastrointestinal tract, in addition to antibiotic exposure, they will also be exposed to an enormous and complex microbial community within the colon. Horizontal gene transfer may then become a significant player in the genome stability and evolution of these bacteria in in vivo situations.

\section{Conclusions}

In the present study, we monitored the genotypic and phenotypic changes of $L$. casei Zhang in a long-term evolution experiment with and without antibiotic (amoxicillin or gentamicin) selection pressure. We found that $L$. casei Zhang has high genome stability even subject to long-term antibiotic stresses. However, whether this is a species-specific or universal characteristic for all probiotic bacteria remains to be explored.

\section{Additional file}

Additional file 1: Table S1. General information of the confirmed SNVs. (DOCX $23 \mathrm{~kb}$ )

\section{Abbreviations}

ALE: Adaptive laboratory evolution; COGs: Clusters of orthologous groups; $E$. coli: Escherichia coli; L. casei Zhang: Lactobacillus casei Zhang; LAB: Lactic acid bacteria; LSM: LAB susceptibility test medium; MICs: Minimum inhibitory concentrations; MRS: de Mann-Rogosa-Sharpe; SNP: Single nucleotide polymorphism; SNV: Single nucleotide variation

\section{Acknowledgments}

JCW thanks Dr. Zhong for advice and assistance with the bioinformatics analysis.

\section{Funding}

This research was supported by the National Natural Science Foundation of China (Grant Nos. 31660457 and 31571815) and the China Agriculture Research System (Grant No. CARS-37).

Availability of data and materials

The individual genomes of 50 strains have been deposited in the National Center for Biotechnology Information (NCBI) Sequence Read Archive (SRA) (http://trace.ncbi.nlm.nih.gov/Traces/sra/sra.cgi) under accession numbers SAMN05735069-SAMN05735118.

\section{Authors' contributions}

HPZ and WYZ conceived the study and participated in its design and coordination. JCW, YYS, HLG, LP, WYH, and LYK carried out the laboratory work. XD and WYZ performed the bioinformatics analysis. WYZ, XD, JCW, and LYK wrote the manuscript. All authors read and approved the final manuscript.

\section{Competing interests}

The authors declare that they have no competing interests.

Consent for publication

Not applicable.

Ethics approval and consent to participate Not applicable.

\section{Publisher's Note}

Springer Nature remains neutral with regard to jurisdictional claims in published maps and institutional affiliations.

Received: 6 December 2016 Accepted: 20 April 2017

Published online: 24 April 2017

\section{References}

1. Lenski RE. Bacterial evolution and the cost of antibiotic resistance. Int Microbiol. 1998;1 (4):265-70.

2. Barrick JE, Yu DS, Yoon SH, Jeong H, Oh TK, Schneider D, Lenski RE, Kim JF. Genome evolution and adaptation in a long-term experiment with Escherichia coli. Nature. 2009;461(7268):1243-47.

3. Russell HL. Bacteriology in its general relation (continued). Am Midl Nat. 1893;27(324):1050-65. 
4. Portnoy VA, Bezdan D, Zengler K. Adaptive laboratory evolution-harnessing the power of biology for metabolic engineering. Curr Opin Biotechnol. 2011;22(4):590-4.

5. Salvetti E, Torriani S, Felis GE. The genus Lactobacillus: ataxonomic update. Probiotics Antimicrob Proteins. 2012;4(4):217-26.

6. Sun Z, Harris HM, McCann A, Guo C, Argimon S, Zhang W, Yang X, Jeffery IB, Cooney JC, Kagawa TF, Liu W, Song Y, Salvetti E, Wrobel A, Rasinkangas P, Parkhill J, Rea MC, O'Sullivan O, Ritari J, Douillard FP, Paul Ross R, Yang R, Briner AE, Felis GE, de Vos WM, Barrangou R, Klaenhammer TR, Caufield PW, Cui Y, Zhang H, O'Toole PW. Expanding the biotechnology potential of lactobacilli through comparative genomics of 213 strains and associated genera. Nat Commun. 2015;6:8322.

7. Ouwehand AC, Salminen S, Isolauri E. Probiotics: an overview of beneficial effects. Antonie Van Leeuwenhoek. 2002;82(1):279-89.

8. Bao WC, Mi ZH, Xu HY, Zheng Y, Kwok LY, Zhang HP, Zhang WY. Assessing quality of Medicago sativa silage by monitoring bacterial composition with single molecule, real-time sequencing technology and various physiological parameters. Sci Rep. 2016;6:28358.

9. Aureli P, Capurso L, Castellazzi AM, Clerici M, Giovannini M, Morelli L, Poli A Pregliasco F, Salvini F, Zuccotti GV. Probiotics and health: an evidence-based review. Pharmacol Res. 2011:63(5):366-76.

10. Song HJ, Kim JY, Jung SA, Kim SE, Park HS, Jeong Y, Hong SP, Cheon JH, Kim WH, Kim HJ, Ye BD, Yang SK, Kim SW, Shin SJ, Kim HS, Sung JK, Kim EY. Effect of probiotic Lactobacillus (Lacidofil(R) cap) for the prevention of antibiotic-associated diarrhea: a prospective, randomized, double-blind, multicenter study. J Korean Med Sci. 2010;25(12):1784-91.

11. Woodford N, Ellington MJ. The emergence of antibiotic resistance by mutation. Clin Microbiol Infect. 2007;13(1):5-18.

12. Drago L, Mattina R, Nicola L, Rodighiero V, De Vecchi E. Macrolide resistance and in vitro selection of resistance to antibiotics in Lactobacillus isolates. J Microbiol. 2011;49(4):651-56.

13. Curragh HJ, Collins MA. High levels of spontaneous drug resistance in Lactobacillus. J Appl Bacteriol. 1992;73(1):31-6.

14. Ya T, Zhang Q, Chu F, Merritt J, Bilige M, Sun T, Du R, Zhang H Immunological evaluation of Lactobacillus casei Zhang: a newly isolated strain from koumiss in Inner Mongolia, China. BMC Immunol. 2008;9(1):68.

15. Wang $Y$, Li Y, Xie J, Zhang Y, Wang J, Sun X, Zhang $H$. Protective effects of probiotic Lactobacillus casei Zhang against endotoxin- and d-galactosamineinduced liver injury in rats via anti-oxidative and anti-inflammatory capacities. Int Immunopharmacol. 2013;15(1):30-7.

16. Zhang W, Yu D, Sun Z, Chen X, Bao Q, Meng H, Hu S, Zhang H. Complete nucleotide sequence of plasmid plca36 isolated from Lactobacillus casei Zhang. Plasmid. 2008;60(2):131-5.

17. Zhang W, Yu D, Sun Z, Wu R, Chen X, Chen W, Meng H, Hu S, Zhang H. Complete genome sequence of Lactobacillus casei Zhang, a new probiotic strain isolated from traditional homemade koumiss in Inner Mongolia, China. J Bacteriol. 2010;192(19):5268-69.

18. Zhang W, Sun Z, Menghe B, Zhang H. Short communication: Single molecule, real-time sequencing technology revealed species- and strain-specific methylation patterns of 2 Lactobacillus strains. J Dairy Sci. 2015;98(5):3020-24.

19. Klare I, Konstabel C, Muller-Bertling S, Reissbrodt R, Huys G, Vancanneyt M, Swings J, Goossens H, Witte W. Evaluation of new broth media for microdilution antibiotic susceptibility testing of Lactobacilli, Pediococci, Lactococci, and Bifidobacteria. Appl Environ Microbiol. 2005;71(12):8982-86.

20. Pena-Miller R, Laehnemann D, Jansen G, Fuentes-Hernandez A, Rosenstiel P, Schulenburg $\mathrm{H}$, Beardmore $\mathrm{R}$. When the most potent combination of antibiotics selects for the greatest bacterial load: the smile-frown transition. PLoS Biol. 2013;11(4):e1001540.

21. Shao YY, Zhang WY, Guo HL, Pan L, Zhang HP, Sun TS. Comparative studies on antibiotic resistance in Lactobacillus casei and Lactobacillus plantarum. Food Control. 2015;50(50):250-58.

22. Hurst LD. The Ka/Ks ratio: diagnosing the form of sequence evolution. Trends Genet. 2002;18(9):486-87.

23. Pirooznia M, Kramer M, Parla J, Goes FS, Potash JB, McCombie WR, Zandi PP. Validation and assessment of variant calling pipelines for next-generation sequencing. Hum Genomics. 2014;8:14.

24. Masiewicz P, Brzostek A, Wolanski M, Dziadek J, Zakrzewska-Czerwinska J. A novel role of the PrpR as a transcription factor involved in the regulation of methylcitrate pathway in Mycobacterium tuberculosis. PLoS One. 2012;7(8):e43651.
25. Morel-Deville F, Fauvel F, Morel P. Two-component signal-transducing systems involved in stress responses and vancomycin susceptibility in Lactobacillus sakei. Microbiology. 1998;144(Pt 10):2873-83.

26. Hamon E, Horvatovich P, Bisch M, Bringel F, Marchioni E, Aoude-Werner D, Ennahar S. Investigation of biomarkers of bile tolerance in Lactobacillus casei using comparative proteomics. J Proteome Res. 2012;11(1):109-18.

27. Denome SA, Elf PK, Henderson TA, Nelson DE, Young KD. Escherichia coli mutants lacking all possible combinations of eight penicillin binding proteins: viability, characteristics, and implications for peptidoglycan synthesis. J Bacteriol. 1999;181(13):3981-93.

28. Goffin C, Ghuysen JM. Biochemistry and comparative genomics of SxxK superfamily acyltransferases offer a clue to the mycobacterial paradox: presence of penicillin-susceptible target proteins versus lack of efficiency of penicillin as therapeutic agent. Microbiol Mol Biol Rev. 2002;66(4):702-38.

29. Suutari M, Laakso S. Temperature adaptation in Lactobacillus fermentum: interconversions of oleic, vaccenic and dihydrosterulic acids. J Gen Microbiol. 1992;138(3):445-50.

30. Boyd DA, Cvitkovitch DG, Bleiweis AS, Kiriukhin MY, Debabov DV, Neuhaus FC, Hamilton IR. Defects in D-alanyl-lipoteichoic acid synthesis in Streptococcus mutans results in acid sensitivity. J Bacteriol. 2000;182(21):6055-65.

31. Broadbent JR, Larsen RL, Deibel V, Steele JL. Physiological and transcriptional response of Lactobacillus casei ATCC 334 to acid stress. J Bacteriol. 2010;192(9):2445-58.

32. Alekshun MN, Levy SB. Molecular mechanism of antibacterial multidrug resistance. Cell. 2007;128(6):1037-50.

33. Lee H, Popodi E, Tang H, Foster PL. Rate and molecular spectrum of spontaneous mutations in the bacterium Escherichia coli as determined by whole-genome sequencing. Proc Natl Acad Sci U S A. 2012;109(41):E2774-83.

34. Schaaper RM, Bond BI, Fowler RG. A.T_-C.G transversions and their prevention by the Escherichia coli mutT and mutHLS pathways. Mol Gen Genet. 1989:219(1):256-62.

\section{Submit your next manuscript to BioMed Central and we will help you at every step:}

- We accept pre-submission inquiries

- Our selector tool helps you to find the most relevant journal

- We provide round the clock customer support

- Convenient online submission

- Thorough peer review

- Inclusion in PubMed and all major indexing services

- Maximum visibility for your research

Submit your manuscript at www.biomedcentral.com/submit
) Biomed Central 